Research Article

\title{
Characteristics of the Soliton Molecule and Lump Solution in the $(2+1)$-Dimensional Higher-Order Boussinesq Equation
}

\author{
Bo Ren \\ Institute of Nonlinear Science, Shaoxing University, Shaoxing 312000, China \\ Correspondence should be addressed to Bo Ren; renbosemail@163.com
}

Received 1 February 2021; Revised 19 March 2021; Accepted 25 March 2021; Published 10 April 2021

Academic Editor: Mohammad Mirzazadeh

Copyright (c) 2021 Bo Ren. This is an open access article distributed under the Creative Commons Attribution License, which permits unrestricted use, distribution, and reproduction in any medium, provided the original work is properly cited.

\begin{abstract}
The soliton molecules, as bound states of solitons, have attracted considerable attention in several areas. In this paper, the $(2+1)$ -dimensional higher-order Boussinesq equation is constructed by introducing two high-order Hirota operators in the usual $(2+1)$ -dimensional Boussinesq equation. By the velocity resonance mechanism, the soliton molecule and the asymmetric soliton of the higher-order Boussinesq equation are constructed. The soliton molecule does not exist for the usual $(2+1)$-dimensional Boussinesq equation. As a special kind of rational solution, the lump wave is localized in all directions and decays algebraically. The lump solution of the higher-order Boussinesq equation is obtained by using a quadratic function. This lump wave is just the bright form by some detail analysis. The graphics in this study are carried out by selecting appropriate parameters. The results in this work may enrich the variety of the dynamics of the high-dimensional nonlinear wave field.
\end{abstract}

\section{Introduction}

The $(2+1)$-dimensional Boussinesq equation can describe the propagation of small-amplitude long waves in shallow water. The physical and dynamical structures of the $(2+1)$ -dimensional Boussinesq equation are investigated by using various methods [1-4]. The $(2+1)$-dimensional Boussinesq equation reads

$$
u_{t t}+\gamma u_{x x}+3 \gamma\left(u^{2}\right)_{x x}-\alpha u_{x x x x}+\mu u_{y y}=0
$$

where $\alpha, \gamma$, and $\mu$ are arbitrary constants. It can be transformed into the Hirota form:

$$
\left(D_{t}^{2}+\gamma D_{x}^{2}-\alpha D_{x}^{4}+\mu D_{y}^{2}\right) f \cdot f=0
$$

with the dependent variable transformation:

$$
u=2(\ln f)_{x x} .
$$

The $(2+1)$-dimensional Boussinesq equation reduces the $(1+1)$-dimensional Boussinesq form with $\mu=0$. The $(1+1)$-dimensional Boussinesq equation includes the "good" Boussinesq form and "bad" Boussinesq form with $\alpha<0$ and $\alpha>0$, respectively [5]. Investigating deeper into properties of this model (1), the extended $(2+1)$-dimensional Boussinesq equations are introduced based on the usual Boussinesq equation (1) $[6,7]$. The topological kink-type soliton solutions of the extended $(2+1)$ -dimensional Boussinesq equation are obtained by the sine-Gordon expansion method [6]. The modified exponential expansion method is applied to the coupled Boussinesq equation [7]. The multisoliton solutions, breather solutions, and rogue waves of the generalized Boussinesq equation are obtained via the symbolic computation method [8] and the polynomial functions in the bilinear form [9]. Generally, seeking exact solutions to nonlinear evolution equations is a vital task in soliton theory. Many methods have been proved effective in finding the exact solutions of the soliton equation [10-12]. By using the extended auxiliary equation method and the extended 
direct algebraic method, the solitary traveling wave solutions and the stability of these solutions are analyzed [10-12]. In this work, we shall study the soliton molecule and lump wave of the higher-order Boussinesq equation by solving the bilinear form of the higher-order Boussinesq equation.

The soliton molecule which is formed by the balance of repulsive and attractive forces between solitons is treated as a boundary state [13]. It was first predicted within the framework of the nonlinear Schrödinger-Ginzburg-Landau equation [14]. Many effects including nonlinear and dispersive effects are a key role in the soliton molecule. The soliton molecule has become a focus of intense research in both experiment and simulation [13-17]. The theoretical frameworks to address the soliton molecule have been introduced [18, 19]. Recently, Lou proposed the velocity resonance mechanism to construct the soliton molecules of the $(1+1)$-dimensional nonlinear systems [20]. The velocity resonance mechanism is one of the useful methods to form the soliton molecule [20]. To balance the nonlinear effects, the high-order dispersive terms may play a key role in the velocity resonance mechanism [21]. The soliton molecule of a variety of integrable systems has been verified with the velocity resonance mechanism: the fifth-order Korteweg-de Vries (KdV) equation $[22,23]$, the modified $\mathrm{KdV}$ equation $[24,25]$, the $(3+1)$ -dimensional Boiti-Leon-Manna-Pempinelli equation [26], and so on [27]. The dynamics between soliton molecules and breather solutions and between soliton molecules and dromions are presented by the velocity resonance mechanism, the Darboux transformation, and the variable separation approach [25-28].

In this paper, we try to construct the $(2+1)$-dimensional higher-order Boussinesq equation which possesses the soliton molecule. The soliton molecule is absent in the usual $(2$ +1 )-dimensional Boussinesq equation. This paper is organized as follows. In Section 2, the soliton molecule and the asymmetric soliton of the $(2+1)$-dimensional higher-order Boussinesq equation are constructed by the velocity reso- nance condition. In Section 3, the lump solution of the higher-order Boussinesq equation is obtained by solving the corresponding Hirota bilinear form. Finally, the conclusions of this paper follow in the last section.

\section{Soliton Molecule for the $(2+1)$-Dimensional Higher-Order Boussinesq Equation}

Based on the bilinear form of the $(2+1)$-dimensional Boussinesq equation, we can construct the higher-order form by introducing the high-order Hirota operators $\left(D_{x}^{6}\right.$ and $\left.D_{y}^{4}\right)$ :

$$
\left(D_{t}^{2}+\gamma D_{x}^{2}-\alpha D_{x}^{4}-\beta D_{x}^{6}+\mu D_{y}^{2}+v D_{y}^{4}\right) f \cdot f=0
$$

where $D$ is the bilinear derivative operator [29]:

$$
\begin{aligned}
D_{x}^{l} D_{y}^{n} D_{t}^{m}(f \cdot g)= & \left(\frac{\partial}{\partial x}-\frac{\partial}{\partial x^{\prime}}\right)^{l}\left(\frac{\partial}{\partial y}-\frac{\partial}{\partial y^{\prime}}\right)^{n} \\
& \left(\frac{\partial}{\partial t}-\frac{\partial}{\partial t^{\prime}}\right)^{m} f(x, y, t) \\
& \left.\cdot g\left(x^{\prime}, y^{\prime}, t^{\prime}\right)\right|_{x} ^{\prime=x, y=y^{\prime}, t^{\prime}=t}
\end{aligned}
$$

Two-soliton solution of the higher-order Boussinesq equation can be calculated as

$$
f=1+\exp \left(\eta_{1}\right)+\exp \left(\eta_{2}\right)+a_{12} \exp \left(\eta_{1}+\eta_{2}\right)
$$

where $\eta_{i}=k_{i} x+l_{i} y+\omega_{i} t+c_{i}(i=1,2)$. By substituting (6) into (4), the phase shift $a_{12}$ and the dispersion relation are written as

$$
\begin{gathered}
a_{12}=\frac{2 \gamma k_{1} k_{2}+2 \mu l_{1} l_{2}+2 v l_{1} l_{2}\left(2 L-3 l_{1} l_{2}\right)-2 \alpha k_{1} k_{2}\left(2 K-3 k_{1} k_{2}\right)-k_{1} k_{2}\left(6 K^{2}-15 k_{1} k_{2} K+8 k_{1}^{2} k_{2}^{2}\right)+2 \omega_{1} \omega_{2}}{2 \gamma k_{1} k_{2}+2 \mu l_{1} l_{2}+2 v l_{1} l_{2}\left(2 L+3 l_{1} l_{2}\right)-2 \alpha k_{1} k_{2}\left(2 K+3 k_{1} k_{2}\right)-k_{1} k_{2}\left(6 K^{2}+15 k_{1} k_{2} K+8 k_{1}^{2} k_{2}^{2}\right)+2 \omega_{1} \omega_{2}} \\
K=k_{1}^{2}+k_{2}^{2} \\
L=l_{1}^{2}+l_{2}^{2} \\
\omega_{i}^{2}+\gamma k_{i}^{2}-\alpha k_{i}^{4}-\beta k_{i}^{6}+\mu l_{i}^{2}+v l_{i}^{4}=0
\end{gathered}
$$




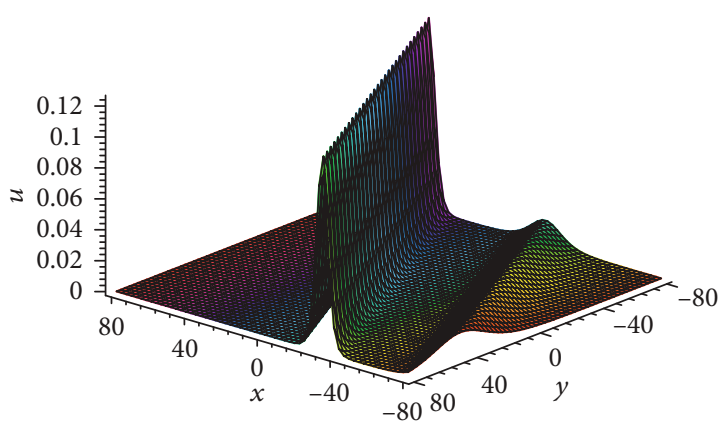

(a)

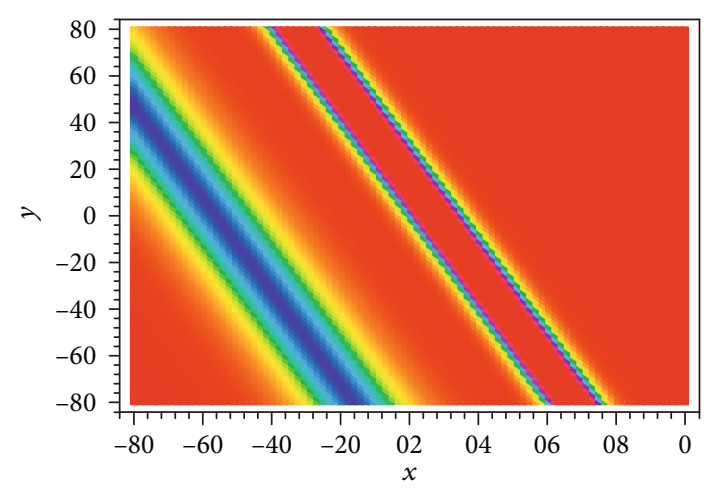

(b)

Figure 1: (a) Soliton molecule of the $(2+1)$-dimensional higher-order Boussinesq equation. (b) Density plot of the corresponding soliton molecule.

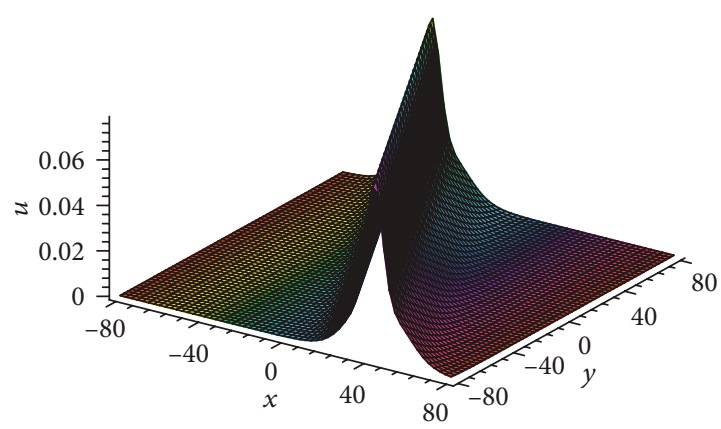

(a)

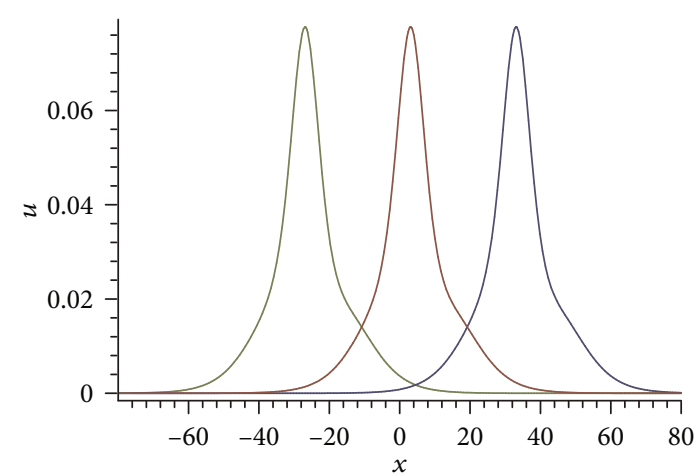

(b)

Figure 2: (a) Asymmetric soliton of the $(2+1)$-dimensional higher-order Boussinesq equation. (b) The wave propagation pattern along the $x$ -axis by selecting different times $t=60, t=0$, and $t=-60$ (from left to right).

The soliton molecule can be constructed with the velocity resonance condition [30]. The velocity resonance condition $\left(k_{i} \neq k_{j}\right)$ reads

$$
\frac{k_{i}}{k_{j}}=\frac{l_{i}}{l_{j}}=\frac{\omega_{i}}{\omega_{j}}=\frac{\sqrt{\alpha k_{i}^{4}+\beta k_{i}^{6}-\gamma k_{i}^{2}-\mu l_{i}^{2}-v l_{i}^{4}}}{\sqrt{\alpha k_{j}^{4}+\beta k_{j}^{6}-\gamma k_{j}^{2}-\mu l_{j}^{2}-v l_{j}^{4}}} .
$$

By solving condition (8), the velocity resonant condition becomes

$$
\begin{aligned}
& k_{j}= \pm \frac{\sqrt{\beta\left(\nu l_{i}^{4}-\alpha k_{i}^{4}-\beta k_{i}^{6}\right)}}{k_{i}^{2}}, \\
& l_{j}= \pm \frac{l_{i} \sqrt{\beta\left(v l_{i}^{4}-\alpha k_{i}^{4}-\beta k_{i}^{6}\right)}}{k_{i}^{3}} .
\end{aligned}
$$

Above velocity resonant condition (9) cannot be obtained while equation (4) is absent in the high-order Hirota operators $D_{x}^{6}$ and $D_{y}^{4}$. A soliton molecule and an asymmetric soliton can be constructed by selecting appropriate parameters in (8) or (9). These phenomena are shown in Figures 1 and 2. We select the same parameters and different phases for Figures 1 and 2. The parameters are

$$
\begin{aligned}
k_{1} & =\frac{1}{2}, \\
k_{2} & =\frac{\sqrt{2}}{8}, \\
l_{1} & =\frac{1}{4}, \\
l_{2} & =\frac{\sqrt{2}}{16}, \\
\alpha & =-\frac{1}{4}, \\
\beta & =1, \\
\gamma & =-1, \\
\mu & =1, \\
\nu & =\frac{1}{2} .
\end{aligned}
$$

The phases of Figures 1 and 2 are $c_{1}=0, c_{2}=10$ and $c_{1}$ 
$=0, c_{2}=1$, respectively. The soliton molecule and the asymmetric soliton are described in Figures 1 and 2. The soliton molecule and the asymmetric soliton can be transformed with each other by selecting different parameters. Two solitons in the molecule have different amplitudes, while two solitons in the molecule possess the same velocity.

\section{Lump Solution of the $(2+1)$-Dimensional Higher-Order Boussinesq Equation}

Lump solutions, which can be considered a kind of rational function solutions, decay polynomially in all directions of space [31-36]. One can construct lump solutions by the Hirota bilinear method and the Darboux transformation [3745]. Lump waves of the high-dimensional nonlinear systems are constructed by solving the Hirota bilinear method [4649]. A symbolic computation approach is one of the useful methods to search the lump wave [31]. The interaction between the lump waves and other complicated waves is presented by the symbolic computation approach [38-43]. In this section, we shall study the dynamics of lump waves by using the symbolic computation approach.

To obtain the lump solution of the $(2+1)$-dimensional higher-order Boussinesq equation, a quadratic function of $f$ is shown as

$$
f=\left(a_{1} x+a_{2} y+a_{3} t\right)^{2}+\left(a_{4} x+a_{5} y+a_{6} t\right)^{2}+a_{7},
$$

where $a_{i}(i=1,2, \cdots, 7)$ are arbitrary constants. By substituting (11) into the Hirota bilinear form (4) and balancing the different powers of $x, y$, and $t$, the parameters are constrained as the following three cases.

Case 1.

$$
\begin{gathered}
a_{1}=\sqrt{\frac{\mu a_{5}^{2}+a_{6}^{2}}{\gamma},} \\
a_{2}=\frac{a_{3} a_{5}}{a_{6}}, \\
a_{4}=\frac{a_{3}}{a_{6}} \sqrt{\frac{\mu a_{5}^{2}+a_{6}^{2}}{\gamma}}, \\
a_{7}=-\frac{3 v a_{5}^{4}\left(a_{3}^{2}+a_{6}^{2}\right)}{a_{6}^{2}\left(\mu a_{5}^{2}+a_{6}^{2}\right)}+\frac{3 \alpha\left(a_{3}^{2}+a_{6}^{2}\right)\left(\mu a_{5}^{2}+a_{6}^{2}\right)}{\gamma^{2} a_{6}^{2}} .
\end{gathered}
$$

The solution of $u$ can be localized in the $(x, y)$-plane with the parameters satisfying

$$
\begin{aligned}
& \mu \nu>0, \\
& a_{7}>0 .
\end{aligned}
$$

Case 2.

$$
\begin{gathered}
a_{1}=\frac{-\mu a_{5}^{2}+a_{6}^{2}}{\gamma}, \\
a_{2}=-a_{5}, \\
a_{4}=\frac{-\mu a_{5}^{2}+a_{6}^{2}}{\gamma}, \\
a_{7}=\frac{6 \alpha\left(\mu a_{5}^{2}-a_{6}^{2}\right)^{2}}{\gamma^{2} a_{6}^{2}}-\frac{6 v a_{5}^{4}}{a_{6}^{2}} .
\end{gathered}
$$

Case 3.

$$
\begin{gathered}
a_{1}=\frac{-\mu a_{5}^{2}+a_{6}^{2}}{\gamma}, \\
a_{3}=-a_{6}, \\
a_{4}=\frac{-\mu a_{5}^{2}+a_{6}^{2}}{\gamma}, \\
a_{7}=\frac{6 \alpha\left(\mu a_{5}^{2}-a_{6}^{2}\right)^{2}}{\gamma^{2} a_{6}^{2}}-\frac{6 v a_{5}^{4}}{a_{6}^{2}} .
\end{gathered}
$$

In order to localize the solution of $u$ in the $(x, y)$-plane for Cases 2 and 3, the parameters should be satisfied:

$$
\alpha\left(\mu a_{5}^{2}-a_{6}^{2}\right)^{2}-v \gamma^{2} a_{5}^{4}>0
$$

Take Case 1 as an example to describe the dynamics of lump waves. By substituting (11) into (3), the lump wave of the $(2+1)$-dimensional higher-order Boussinesq equation in Case 1 is generated:

$$
u=\frac{4\left(a_{3}^{2}+a_{6}^{2}\right)\left(\mu a_{5}^{2}+a_{6}^{2}\right)}{\gamma a_{6}^{2} f}-\frac{8\left(a_{3}^{2}+a_{6}^{2}\right)^{2}\left(\mu a_{5}^{2}+a_{6}^{2}\right)^{2} x^{2}}{\gamma^{2} a_{6}^{4} f^{2}} .
$$

To describe the lump wave of the $(2+1)$-dimensional higher-order Boussinesq equation, the parameters are selected as

$$
\begin{aligned}
& \alpha=1, \\
& \gamma=1, \\
& a_{3}=1, \\
& a_{5}=3, \\
& a_{6}=2, \\
& \mu=1, \\
& \nu=\frac{1}{2} .
\end{aligned}
$$

The spatiotemporal structure and the density of a lump wave are described in Figures 3(a) and 3(b), respectively. 


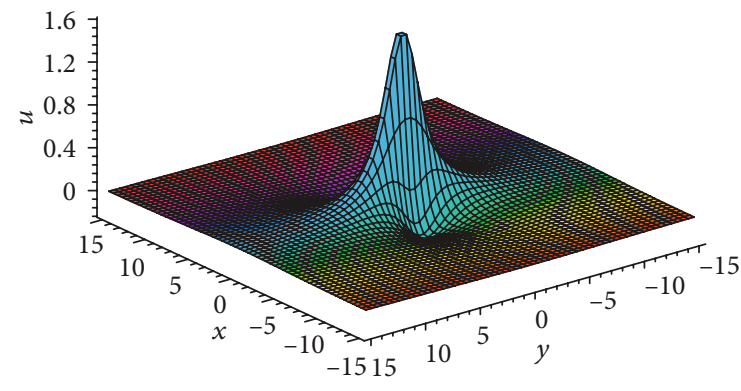

(a)

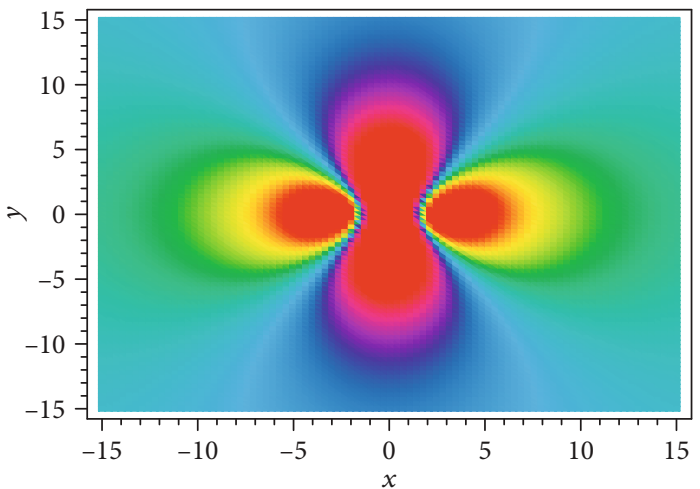

(b)

Figure 3: (a) The three-dimensional plot of a lump wave of the $(2+1)$-dimensional higher-order Boussinesq equation with the parameters in (18). (b) The corresponding density plot.

The critical points of the lump wave are solved:

$$
\begin{aligned}
& \frac{\partial u(x, y, t)}{\partial x}=0, \\
& \frac{\partial u(x, y, t)}{\partial y}=0 .
\end{aligned}
$$

By solving above condition (19), we find that the function $u$ reaches the maximum value at the point $\left(0,-\left(a_{6} / a_{5}\right) t\right)$ and the minimum values at two points $( \pm(3$ $\left.\left.\sqrt{\alpha\left(\mu a_{5}^{2}+a_{6}^{2}\right)^{2}-v \gamma^{2} a_{5}^{2}}\right) /\left(\sqrt{\gamma}\left(\mu a_{5}^{2}+a_{6}^{2}\right)\right),-\left(a_{6} / a_{5}\right) t\right) . \quad$ By substituting above three points values into (17), the maximum and minimum values of the function $u$ are $(4 \gamma$ $\left.\left(\mu a_{5}^{2}+a_{6}^{2}\right)^{2}\right) /\left(3\left(\alpha\left(\mu a_{5}^{2}+a_{6}^{2}\right)^{2}-v \gamma^{2} a_{5}^{2}\right)\right)$ and $-\left(\gamma\left(\mu a_{5}^{2}+a_{6}^{2}\right)^{2}\right) /$ $\left(6\left(\alpha\left(\mu a_{5}^{2}+a_{6}^{2}\right)^{2}-v \gamma^{2} a_{5}^{2}\right)\right)$, respectively. The value of the maximum point is bigger than zero due to $a_{7}>0$. The ratio between the maximum and minimum amplitudes is 8 . The lump wave of the higher-order Boussinesq equation is just the bright form by the above detail analysis.

\section{Conclusion}

In summary, the soliton molecule and lump solution of the $(2+1)$-dimensional higher-order Boussinesq equation are studied by solving the Hirota bilinear form (4). The soliton molecule and the asymmetric soliton are obtained by the velocity resonance mechanism. The lump solution can be derived by using a positive quadratic function. The lump wave of the higher-order Boussinesq equation is just the bright form after some detail analysis. Figures 1-3 show the dynamics of the soliton molecule and lump wave by putting suitable parameters. The soliton molecule and the asymmetric soliton can be transformed with each other by selecting different phases. The soliton molecule and the asymmetric soliton cannot be derived in the $(2+1)$-dimensional Boussinesq equation (1).

In this paper, the $(2+1)$-dimensional higher-order Boussinesq equation is constructed by introducing the high-order Hirota bilinear operators $D_{x}^{6}$ and $D_{y}^{4}$ based on the usual $(2+1)$-dimensional Boussinesq equation. Similar to introducing the high-order Hirota bilinear operator procedure, we propose one equation

$$
\left(D_{t}^{2}+\gamma D_{x}^{2}-\sum_{i=1}^{n}\left(\alpha_{i} D_{x}^{2+2 i}\right)+\sum_{j=1}^{m}\left(\beta_{j} D_{y}^{2 j}\right)\right) f \cdot f=0,
$$

with $\alpha_{i}$ and $\beta_{j}$ being arbitrary constants. The soliton molecule and lump wave of (20) are worthy of study by the velocity resonance mechanism and the symbolic computation approach. Rogue waves are unexpectedly high-amplitude single waves that have been reported by using the Hirota bilinear method [50, 51]. These nonlinear excitations of (20) are valuable to increase understanding of the phenomena between different nonlinear waves.

\section{Data Availability}

The datasets supporting the conclusions of this article are included in the article.

\section{Conflicts of Interest}

The authors declare that they have no conflict of interest.

\section{Acknowledgments}

This work is supported by the National Natural Science Foundation of China (No. 11775146).

\section{References}

[1] F. Özpinar, H. M. Baskonus, and H. Bulut, "On the complex and hyperbolic structures for the $(2+1)$-dimensional Boussinesq water equation," Entropy, vol. 17, no. 12, pp. 82678277, 2015.

[2] H. Zhang, X. Meng, J. Li, and B. Tian, "Soliton resonance of the -dimensional Boussinesq equation for gravity water waves," Nonlinear Analysis: Real World Applications, vol. 9, no. 3, pp. 920-926, 2008. 
[3] A. S. A. Rady, E. S. Osman, and M. Khalfallah, "On soliton solutions of the $(2+1)$-dimensional Boussinesq equation," Applied Mathematics and Computation, vol. 219, no. 8, pp. 3414-3419, 2012.

[4] X. B. Wang, S. F. Tian, C. Y. Qin, and T. T. Zhang, "Characteristics of the breathers, rogue waves and solitary waves in a generalized (2+1)-dimensional Boussinesq equation," Europhysics Letters, vol. 115, no. 1, article 10002, 2016.

[5] J. G. Rao, Y. B. Liu, C. Qian, and J. S. He, "Rogue waves and hybrid solutions of the Boussinesq equation," Zeitschrift Fur Naturforschung Section A, vol. 72, no. 4, pp. 307-314, 2017.

[6] J. L. García Guirao, H. M. Baskonus, and A. Kumar, "Regarding new wave patterns of the newly extended nonlinear $(2+1)$ dimensional Boussinesq equation with fourth order," Mathematics, vol. 8, no. 3, p. 341, 2020.

[7] T. A. Sulaiman, H. Bulut, A. Yokus, and H. M. Baskonus, "On the exact and numerical solutions to the coupled Boussinesq equation arising in ocean engineering," Indian Journal of Physics, vol. 93, no. 5, pp. 647-656, 2019.

[8] Y. L. Ma, "N-solitons, breathers and rogue waves for a generalized Boussinesq equation," International Journal of Computer Mathematics, vol. 97, no. 8, pp. 1648-1661, 2020.

[9] Y. L. Ma and B. Q. Li, "Analytic rogue wave solutions for a generalized fourth-order Boussinesq equation in fluid mechanics," Mathematical Methods in the Applied Sciences, vol. 42, no. 1, pp. 39-48, 2019.

[10] A. R. Seadawy, "Stability analysis for two-dimensional ionacoustic waves in quantum plasmas," Physics of Plasmas, vol. 21, no. 5, article 052107, 2014.

[11] A. R. Seadawy, "Travelling-wave solutions of a weakly nonlinear two-dimensional higher-order Kadomtsev-Petviashvili dynamical equation for dispersive shallow-water waves," The European Physical Journal Plus, vol. 132, no. 29, pp. 1-13, 2017.

[12] Y. S. Özkan, E. Yaşar, and A. R. Seadawy, "A third-order nonlinear Schrödinger equation: the exact solutions, groupinvariant solutions and conservation laws," Journal of Taibah University for Science, vol. 14, no. 1, pp. 585-597, 2020.

[13] L. Gui, P. Wang, Y. Ding et al., "Soliton molecules and multisoliton states in ultrafast fibre lasers: intrinsic complexes in dissipative systems," Applied Sciences, vol. 8, no. 2, p. 201, 2018.

[14] B. A. Malomed, "Bound solitons in the nonlinear SchrödingerGinzburg-Landau equation," Physical Review A, vol. 44, no. 10, pp. 6954-6957, 1991.

[15] B. Ortaç, A. Zaviyalov, C. K. Nielsen et al., "Observation of soliton molecules with independently evolving phase in a modelocked fiber laser," Optics Letters, vol. 35, no. 10, pp. 15781580, 2010.

[16] M. Stratmann, T. Pagel, and F. Mitschke, "Experimental observation of temporal soliton molecules," Physical Review Letters, vol. 95, no. 14, article 143902, 2005.

[17] K. Krupa, K. Nithyanandan, U. Andral, P. Tchofo-Dinda, and P. Grelu, "Real-time observation of internal motion within ultrafast dissipative optical soliton molecules," Physical Review Letters, vol. 118, no. 24, article 243901, 2017.

[18] U. Al Khawaja, "Stability and dynamics of two-soliton molecules," Physical Review E, vol. 81, no. 5, article 056603, 2010.

[19] L. C. Crasovan, Y. V. Kartashov, D. Mihalache, L. Torner, Y. S. Kivshar, and V. M. Pérez-García, "Soliton molecules: robust clusters of spatiotemporal optical solitons," Physical Review E, vol. 67, no. 4, article 046610, 2003.
[20] S. Y. Lou, "Soliton molecules and asymmetric solitons in three fifth order systems via velocity resonance," Journal Physiscs Communications, vol. 4, no. 4, article 041002, 2020.

[21] D. H. Xu and S. Y. Lou, "Dark soliton molecules in nonlinear optics," Acta Physica Sinica, vol. 69, no. 1, article 014208, 2020.

[22] Z. Zhang, S. X. Yang, and B. Li, "Soliton molecules, asymmetric solitons and hybrid solutions for (2+1)-dimensional fifthorder KdV equation," Chinese Physics Letters, vol. 36, no. 12, article 120501, 2019.

[23] B. Ren and J. Lin, "Soliton molecules, nonlocal symmetry and $\mathrm{CRE}$ method of the KdV equation with higher-order corrections," Physica Scripta, vol. 95, no. 7, article 075202, 2020.

[24] B. Ren, J. Lin, and P. Liu, "Soliton molecules and the CRE method in the extended mKdV equation," Communications in Theoretical Physics, vol. 72, no. 5, article 055005, 2020.

[25] Z. Zhang, X. Yang, and B. Li, "Soliton molecules and novel smooth positons for the complex modified KdV equation," Applied Mathematics Letters, vol. 103, article 106168, 2020.

[26] C. J. Cui, X. Y. Tang, and Y. J. Cui, "New variable separation solutions and wave interactions for the $(3+1)$-dimensional Boiti-Leon-Manna-Pempinelli equation," Applied Mathematics Letters, vol. 102, article 106109, 2020.

[27] B. Ren, "Painlevé analysis, soliton molecule, and lump solution of the higher-order Boussinesq equation," Advances in Mathematical Physics, vol. 2021, Article ID 6687632, 6 pages, 2021.

[28] Z. Yan and S. Lou, "Soliton molecules in Sharma-Tasso-OlverBurgers equation," Applied Mathematics Letters, vol. 104, article 106271, 2020.

[29] R. Hirota, The Direct Method in Soliton Theory, Cambridge University Press, Cambridge, 2004.

[30] B. Ren and J. Lin, "D'Alembert wave and soliton molecule of the modified Nizhnik-Novikov-Veselov equation," The European Physical Journal Plus, vol. 136, no. 1, p. 123, 2021.

[31] W. X. Ma, "Lump solutions to the Kadomtsev-Petviashvili equation," Physics Letters A, vol. 379, no. 36, pp. 1975-1978, 2015.

[32] Y. F. Hua, B. L. Guo, W. X. Ma, and X. Lü, "Interaction behavior associated with a generalized $(2+1)$-dimensional Hirota bilinear equation for nonlinear waves," Applied Mathematical Modelling, vol. 74, pp. 184-198, 2019.

[33] X. W. Jin and J. Lin, "Rogue wave, interaction solutions to the KMM system," Journal of Magnetism and Magnetic Materials, vol. 502, article 166590, 2020.

[34] C. Y. Qin, S. F. Tian, X. B. Wang, and T. T. Zhang, "On breather waves, rogue waves and solitary waves to a generalized $(2+1)$-dimensional Camassa-Holm-Kadomtsev-Petviashvili equation," Communications in Nonlinear Science and Numerical Simulation, vol. 62, pp. 378-385, 2018.

[35] X. W. Yan, S. F. Tian, M. J. Dong, L. Zhou, and T. T. Zhang, "Characteristics of solitary wave, homoclinic breather wave and rogue wave solutions in a (2+1)-dimensional generalized breaking soliton equation," Computers Mathematics with Applications, vol. 76, no. 1, pp. 179-186, 2018.

[36] C. Q. Dai, J. Liu, Y. Fan, and D. G. Yu, "Two-dimensional localized Peregrine solution and breather excited in a variable-coefficient nonlinear Schrödinger equation with partial nonlocality," Nonlinear Dynamics, vol. 88, no. 2, pp. 1373-1383, 2017.

[37] S. Lou and J. Lin, "Rogue waves in nonintegrable KdV-type systems," Chinese Physics Letters, vol. 35, no. 5, article 050202, 2018 
[38] B. Ren, W. X. Ma, and J. Yu, "Rational solutions and their interaction solutions of the $(2+1)$-dimensional modified dispersive water wave equation," Computers Mathematics with Applications, vol. 77, no. 8, pp. 2086-2095, 2019.

[39] B. Ren, W. X. Ma, and J. Yu, "Characteristics and interactions of solitary and lump waves of a $(2+1)$-dimensional coupled nonlinear partial differential equation," Nonlinear Dynamics, vol. 96, no. 1, pp. 717-727, 2019.

[40] X. Zhang, Y. Chen, and X. Tang, "Rogue wave and a pair of resonance stripe solitons to KP equation," Computers Mathematics with Applications, vol. 76, no. 8, pp. 1938-1949, 2018.

[41] L. Huang, Y. Yue, and Y. Chen, "Localized waves and interaction solutions to a $(3+1)$-dimensional generalized KP equation," Computers Mathematics with Applications, vol. 76, no. 4, pp. 831-844, 2018.

[42] J. P. Yu and Y. L. Sun, "Lump solutions to dimensionally reduced Kadomtsev-Petviashvili-like equations," Nonlinear Dynamics, vol. 87, no. 2, pp. 1405-1412, 2017.

[43] B. Ren, J. Lin, and Z. M. Lou, "Consistent Riccati expansion and rational solutions of the Drinfel'd-Sokolov- Wilson equation," Applied Mathematics Letters, vol. 105, article 106326, 2020.

[44] N. Akhmediev, A. Ankiewicz, and J. M. Soto-Crespo, "Rogue waves and rational solutions of the nonlinear Schrödinger equation," Physical Review E, vol. 80, no. 2, article 026601, 2009.

[45] L. Kaur and A. M. Wazwaz, "Lump, breather and solitary wave solutions to new reduced form of the generalized BKP equation," International Journal of Numerical Methods for Heat Fluid Flow, vol. 29, no. 2, pp. 569-579, 2019.

[46] K. Hosseini, M. Samavat, M. Mirzazadeh, W. X. Ma, and Z. Hammouch, "A new (3+1)-dimensional Hirota bilinear equation: its Bäcklund transformation and rational-type solutions," Regular and Chaotic Dynamics, vol. 25, no. 4, pp. 383391, 2020.

[47] K. Hosseini, R. Ansari, R. Pouyanmehr, F. Samadani, and M. Aligoli, "Kinky breather-wave and lump solutions to the (2+1)-dimensional Burgers equations," Analysis and Mathematical Physics, vol. 10, no. 65, p. 120, 2020.

[48] Y. L. Ma and B. Q. Li, "Mixed lump and soliton solutions for a generalized (3+1)-dimensional Kadomtsev-Petviashvili equation," AIMS Mathematics, vol. 5, no. 2, pp. 1162-1176, 2020.

[49] L. Kaur and A. M. Wazwaz, "Dynamical analysis of lump solutions for $(3+1)$ dimensional generalized KP-Boussinesq equation and its dimensionally reduced equations," Physica Scripta, vol. 93, no. 7, article 075203, 2018.

[50] Y. L. Ma and B. Q. Li, "Interactions between soliton and rogue wave for a $(2+1)$-dimensional generalized breaking soliton system: hidden rogue wave and hidden soliton," Computers Mathematics with Applications, vol. 78, no. 3, pp. 827-839, 2019.

[51] Y. L. Ma, "Interaction and energy transition between the breather and rogue wave for a generalized nonlinear Schrödinger system with two higher-order dispersion operators in optical fibers," Nonlinear Dynamics, vol. 97, no. 1, pp. 95-105, 2019. 